\title{
Revisiting the Cloud/s: Interfacing open weather data for live audio-visual performance
}

\author{
Julian Stadon \\ University of Hertfordshire \\ 223 Bromyard House, Bromyard Ave. \\ London W3 7BN, UK \\ j.stadon@herts.ac.uk
}

\begin{abstract}
What does the Anthropocene look like? Should it be described as a singular decisive moment, or a series of banal everyday events? How implicit can an individual feel within such an evolving and accumulative system? This paper explores such questions, through the lens of performative digital arts practice, presenting projects that interface the weather with digital systems, remediating atmospheric data with audio-visual performance tools, to reconfigure how we access and therefore analyse such phenomena. Weather has traditionally been perceived as rather banal, except for particularly significantly abnormal events. Today in a media landscape of fake news, attacks on scientific research and climate change denial, this paper argues that new methods are required for how we interface humans with weather data, be it archival, real time or predictive. Digital media offers novel solutions for the reconfiguring of weather data signification, that allow for a deepening of discourses relating to the weather, through a range of interactive and locational scenarios.
\end{abstract}

Audio-visual performance. Weather data. Anthropocene. Mixed reality. Live coding.

\section{INTRODUCTION}

This paper argues that greater public awareness about the Anthropocene can be established and evolved, through using live weather data as a realtime input for performative, artistic audio-visual environments. Introducing the research of the art collective Swan Panda, it will discuss recently developed artistic methods for integrating real-time weather data into telematics live audio-visual performance, that aim to reconfigure the ways in which we represent, access and respond to the information produced by, and about weather and in turn, reconfigure how the public perceives it. Both weather and digital systems are inherently disruptive and adaptive, and also both are temporal and locational, with the representation of both are mediated by social, political and economic agendas.

By critically engaging with weather data, Swan Panda place an emphasis on the Anthropocene and how this can be represented in artistic practice. This research practice aims to contribute new knowledge to the important project of imagining new and critical forms of art production in the age of the Anthropocene, which directly engage the viewer with the topics that surround it.

\section{FRAMEWORK}

One must enter the catastrophe to reap its benefits without suffering its drawbacks. You cannot prohibit the catastrophe, you must surf it (Virilio 1998).

What does the Anthropocene look like? Should it be described as a singular decisive moment, or a series of banal everyday events? How implicit can an individual feel within such an evolving and accumulative system? With recent growth in natural and human caused catastrophes, there seems to be need for refinement and redefining of how art and culture responds to such questions. The artist collective Swan Panda explores the phenomenon of catastrophe, particularly The Anthropocene, through the lens of performative digital arts practice, presenting projects that interface the weather with digital systems, remediating atmospheric data with audio-visual performance tools, to reconfigure how we access and therefore, analyse such phenomena.

Since industrialisation, weather has been perceived in the general public as rather banal, except for particularly abnormal and significant events. As such events tend to be catastrophic by nature, at each event, new methods or representation and 
response are required to productively respond to them. Henri Bergson claimed that we have to change our way of thinking when facing such new events and this can also be contextualised to discuss how artists represent these phenomena. Like most 'new' art forms:

[T]he idea that, for a new object, we might have to create a new concept, perhaps a new way of thinking, is deeply repugnant to us (Bergson 1907).

Much of the motivation for this research stems from an artistic interpretation of common perceptions by the public and the media in relation to The Anthropocene, with the goal to create objects of convergence between natural and digital systems that both co-exist and evolve symbiotically. Swan Panda formed in 2014 as a transdisciplinary research collaborative with a general mandate to highlight and reconfigure public understandings of environmental phenomena, such as climate change and explore new ways to build empathy for our impact on such phenomena, using performance, intermediality and networked digital technology as prosthetic devices to connect our human condition with our on-going impact on ecological processes and raise awareness about the Anthropocene. Consisting of Julian Stadon and Jorge Ramirez, Swan Panda use a combination of open hardware, software and live programmed audio-visual compositions, in order to challenge contemporary weather data aesthetics, using a range of aesthetic conventions such as glitch aesthetics, gif animation, sampling and live coding, also known as algorave, to do so (McLean \& Dean 2018).

\section{METHOD}

The performative practical method of Swan Panda involves taking atmospheric data from single/ multiple locations and integrating it into live coded musical compositions that are then projected into discrete immediate environments or streamed live, to telematic spatial scenarios. These displaced physical locations are converged and augmented though datafication and fed back into each other, through audio and visual interfacing. The resulting sound and imagery refer to a range of phases within our interpretation of post natural, post biological and post Internet aesthetics.

Zartman and Berman conceptualise negotiations in terms of three main phases (Zartman 1982):

(i) The diagnostic phase.

(ii) The formula phase

(iii) The detail phase.

As Skovdin points out in relation to this concept, within the subjective discourses surrounding The
Anthropocene, generally there is a dichotomy of approaches to seeking information about the topic:

1. Information and knowledge about the nature of the problem in question.

2. Information about their opponents'/partners' strategy and payoff.

In a rational method, both approaches are equally important in forming a rational negotiating strategy in both integrative and distributive bargaining. However, in order to argue effectively one way or the other, it is not necessary apply both types of information equally. (Skovdin 2000) This is where much of the conflict (in media, climate change denial and acceptance for example) evolved. In order to present a holistic articulation of human interactions with and impact on weather and natural systems, Swan Panda has adopted a more syncretic approach that converges several points of view, aiming to create a form of chimerical, transcendental output, that is in itself, a new biodigitally convergent object.

Specifically, this method operates as follows:

1. Specific locations are chosen to collect weather data from, based on the location of both artists, for example in our first performance in 2016, one artist was in Corfu and one was in Mexico City so these two locations were chosen, as the weather is considered as the primary medium for this artistic collective and therefore, should be subjectively specific to each member of the collaborative, much like other mediums would be in more traditional collaborations.

2. Weather data is then obtained, using a combination of a bespoke weather sensing station and using the Weather Underground JSON API. This allows for a combination of online, offline and also local and remote weather streams to be simultaneously fed into the live coded composition.

3. This data is then fed into 2 separate systems that communicate by sending an OSC IP and port message. One system uses the JSON weather stream with Supercollider and the other uses the bespoke weather sensor stream, porting this into Max/MSP. The sound composition consists of live coded generative/ mediated soundscapes in Supercollider, mixed with samples that are played via a Max/MSP patch, both running through a 2-track mixer.

4. A data loop is then created, by using microphone inputted feedback on the Supercollider system and an application written for Max/MSP that also implements a GIF video player.

5. The GIF visualizer is embedded within the Max/MSP patch and uses the accumulative 
composited audio feed, to live compose and cycle through over 400 sample GIFs, all created by the artists. These visual samples are all inspired by The Anthropocene and consist of a combination of filmed and found video and GIF footage, 3D animation, glitch renderings, remixed vaporwave animations, bio/digital phenomena and other postbiological, post-internet aesthetics-based animations relating to The Anthropocene.

6. In the physical setting for the performance, two projections are displayed, with one showing the live coding in Supercollider taking place and the Max/MSP GIF visualisation mix shown on the other

This method can be performed with either/or artist/s present or telepresent and also has the versatility to operate with or without either the weather sensor or JSON weather stream.

\section{IMPLEMENTATION: PERFORMANCES}

\subsection{Fukuoka/Tokyo (2015)}

After forming on the beaches of Pula and the streets of Trieste and Venice in 2014, Swan Panda first performed publicly at the prestigious Fukuoka City Museum and the cult underground club, Soup in Tokyo as part of the Algorave Japan Tour (Bell 2015) and the Data Body as Artifact Exhibition (Stadon 2015). Due to both artists being in the same location, this initial iteration used data from a bespoke weather sensor within the walls of the venues that ported into a real-time Supercollider and Max/MSP procedural composition. These initial live compositions were rather erratic and chaotic, both audibly and visually.

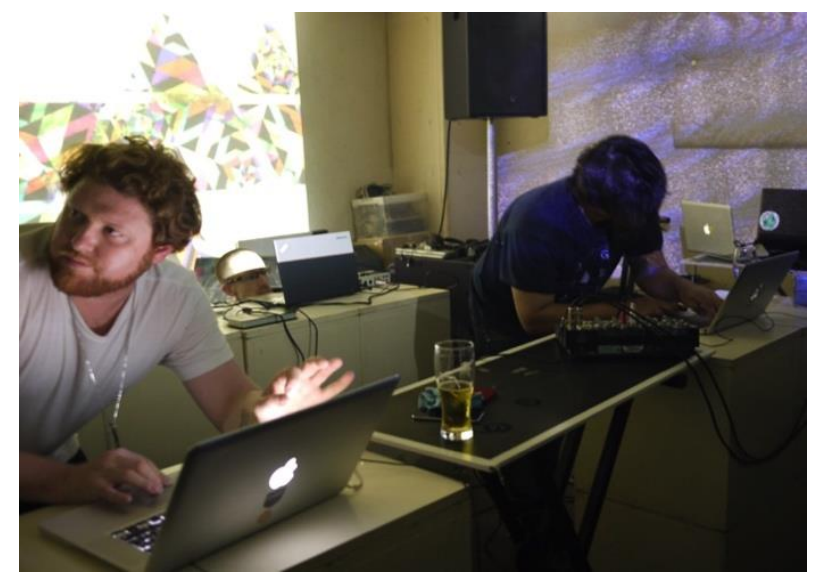

Figure 1: Swan Panda set at Soup, Tokyo.

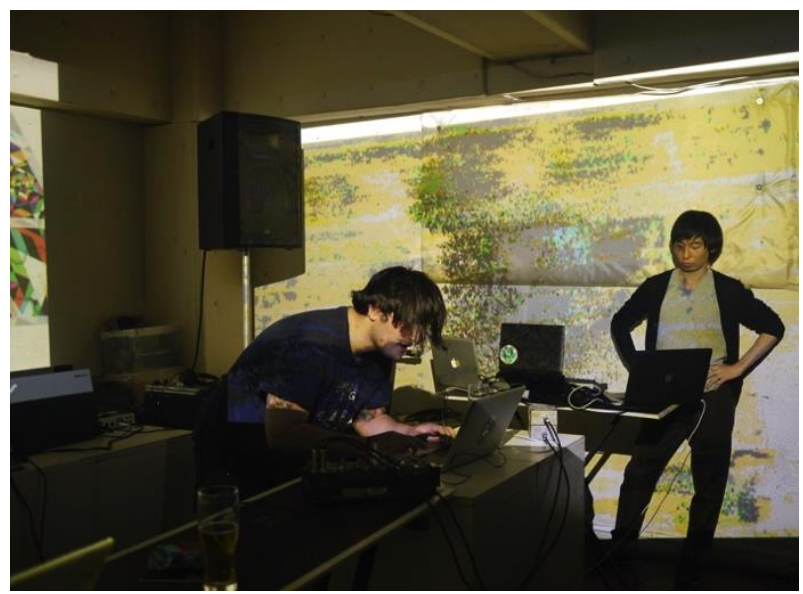

Figure 2: Swan Panda set at Soup, Tokyo.

\subsection{Corfu (2016 \& 2017)}

For the last two years, Swan Panda has been invited to play at the Polytechno Club, as part of AVFest and the Taboo - Transgression Transcendence in Art and Science Conference, held by the Ionian University in Corfu, Greece (Honorato 2016 \& 2017). These performances saw a shift towards remote telematic performance, with Stadon playing live at the venues in Corfu and Ramirez connecting through via a variety of live streams, including HD video and audio, along with a JSON weather stream, using the Weather Underground API. In 2016 Ramirez performed telematically from Mexico City and in 2017 this set up was repeated, with him performing from Guadalajara. In both cases the performance took place physically at the Polytechno venue and also online through the Livestream HD streaming platform, displaying the live coding in Supercollider taking place on one projector and the Max/MSP GIF visualizer on a second projector.

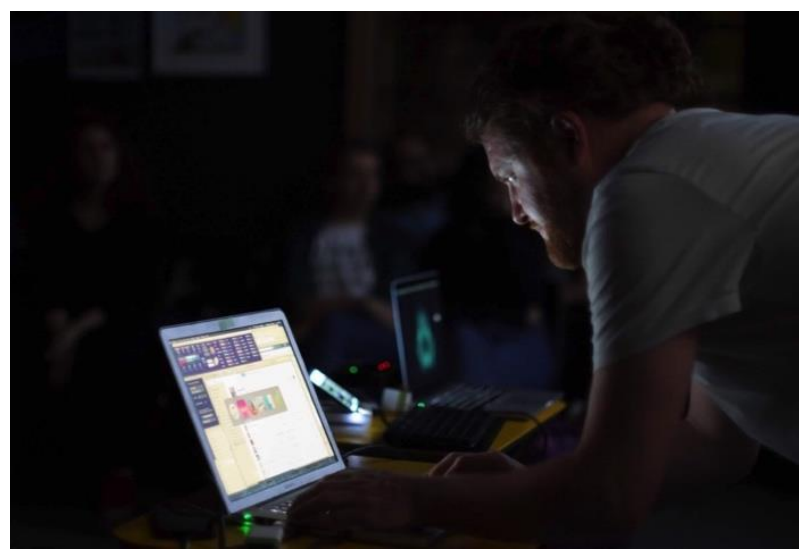

Figure 3: Swan Panda set at Polytehno, Corfu. 


\subsection{Guadalajara (2017)}

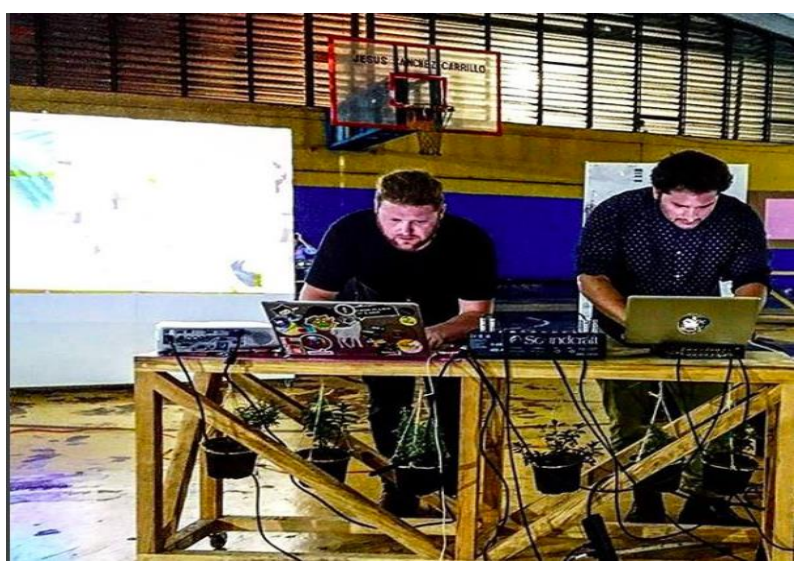

Figure 4: Swan Panda set at Gimnasio Gaston Carreaga, Guadalajara.

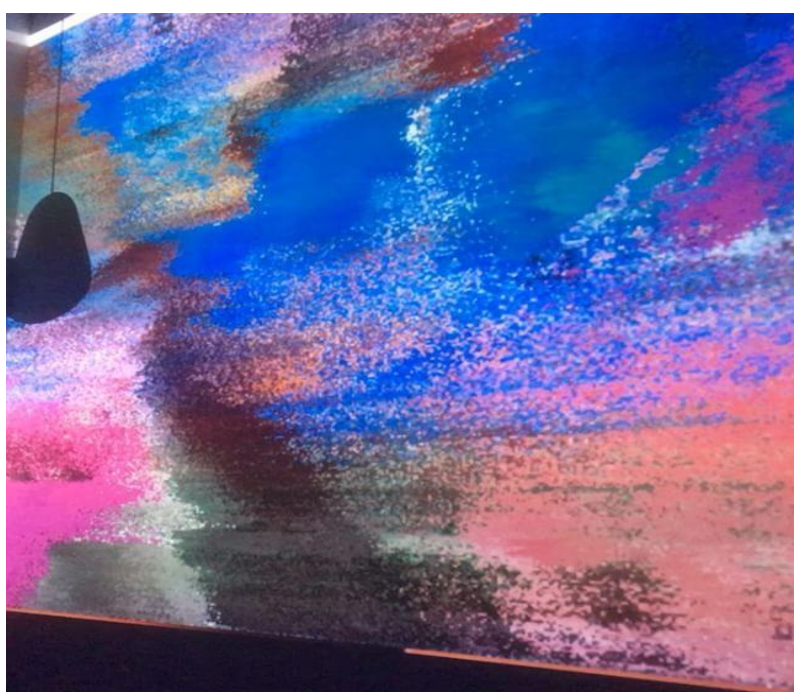

Figure 5: Swan Panda Set Detail.

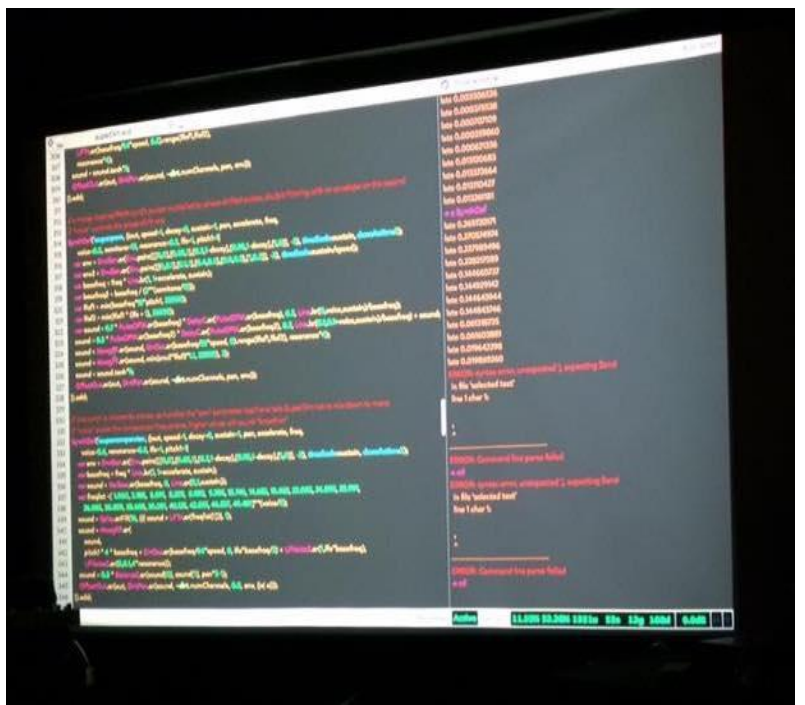

Figure 6: Swan Panda Set Detail.
Swan Panda was invited to play at the Gimnasio Gaston Carreagá in Guadalajara but the Cuidad Creativa Digital Guadalajara CCD Lab (2017) launch. This performance brought both artists back together in the same physical location for the first time in two years, at a venue with a huge acoustic resonance, a gymnasium with multiple curvatures on the roof. This lead to the artistic decision to transition into a more drone-based composition to better accommodate the acoustics of the venue and this sonic aesthetic style has remained in all subsequent performances.

\subsection{Lisbon (2017)}

Following two years of successful performances in Corfu, Swan Panda were invited to perform and speak at the FACTT - Festival Art \& Science Trans-disciplinary and Trans-national in Lisbon. This performance took place at the iconic Museum of Art, Architecture and Technology (MAAT) in the old power station venue. Again, both artists were able to perform together in person and data was taken from the locations of Lisbon and São Luis in Southern Portugal, where the artists undertook a research residency at Cultivamos Cultura in the weeks leading up to the performance. Like Guadaljara, the venue had a venue unique acoustic quality and due to this, a droning sonic aesthetic was implemented once more, in part due to the rather static weather conditions at both locations.

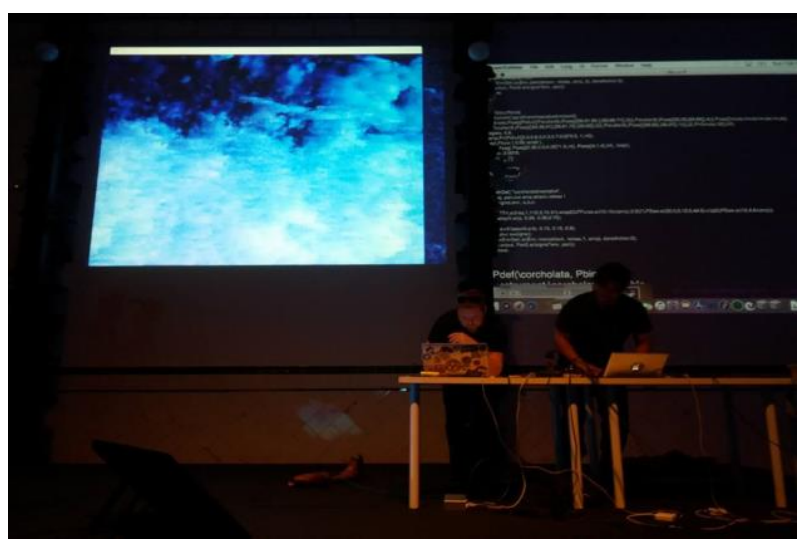

Figure 7: Swan Panda Set at MAAT, Lisbon.

\section{CONCLUSION}

The performative practice of Swan Panda serves as an example of how art can reconfigure and disrupt traditional representational methods for weather and atmospheric conditions in ways that articulate the symbiotic relationships humans have with such systems, and also how this is all now embedded in networked digital systems. As cultural producers, artists have a responsibility to engage with such systems and weave them into the wider fabric of society. 


\section{REFERENCES}

Bell, R. (2015) Alograve Japan Tour, 2015. http://www.renickbell.net/doku.php?id=japan algor ave tour oct. 3-10 (retrieved 20 March 2018).

Bergson, H. (1907) Creative Evolution. Dover Publications, New York.

Honorato, D. (2016 \& 2017) Taboo Transgression-Transcendence in Art and Science Conference. https://avarts.ionio.gr/ttt/2016/en/ \& https://avarts.ionio.gr/ttt/2017/en/ (retrieved 20 March 2018).

McLean, A., and Dean, T. (2018) The Oxford Handbook of Algorithmic Music. Oxford University Press, New York.
Skodvin, T. (2000) Structure and Agent in the Scientific Diplomacy of Climate Change. Kluwer Academic Publishers, Dordrecht.

Stadon, J. (2015) Data Body as Artifact Exhibition. https://marart.org/\#/databodyasartifact (retrieved 20 March 2018).

Virilio, P. (1998) Surfing the Accident. In Brouwer \& Mulder (eds), The Art of the Accident. V2_Publishing, Rotterdam.

Zartman, I. W. and M. R. Berman (1982), The Practical Negotiator. New Haven and London: Yale University Press. 